\title{
Global Dynamics of an SIRS Epidemic Model with Distributed Delay on Heterogeneous Network
}

\author{
Qiming Liu, ${ }^{1}$ Baochen Li, ${ }^{2}$ and Meici Sun ${ }^{1}$ \\ ${ }^{1}$ Institute of Applied Mathematics, Shijiazhuang Mechanical Engineering College, Shijiazhuang, China \\ ${ }^{2}$ Department of Science and Research, Shijiazhuang Mechanical Engineering College, Shijiazhuang 050003, China \\ Correspondence should be addressed to Qiming Liu; lqmmath@163.com
}

Received 1 November 2016; Revised 21 January 2017; Accepted 31 January 2017; Published 26 February 2017

Academic Editor: Leonid Shaikhet

Copyright (c) 2017 Qiming Liu et al. This is an open access article distributed under the Creative Commons Attribution License, which permits unrestricted use, distribution, and reproduction in any medium, provided the original work is properly cited.

\begin{abstract}
A novel epidemic SIRS model with distributed delay on complex network is discussed in this paper. The formula of the basis reproductive number $R_{0}$ for the model is given, and it is proved that the disease dies out when $R_{0}<1$ and the disease is uniformly persistent when $R_{0}>1$. In addition, a unique endemic equilibrium for the SIRS model exists when $R_{0}>1$, and a set of sufficient conditions on the global attractiveness of the endemic equilibrium for the system is given.
\end{abstract}

\section{Introduction}

Following the seminal work on small-world network by Watts and Strogatz [1], and the scale-free network, in which the probability of $p(k)$ for any node with $k$ links to other nodes is distributed according to the power law $p(k)=C k^{-\gamma}(2<$ $\gamma \leq 3$ ), suggested by Barabási and Albert [2], the spreading of epidemic disease on heterogeneous network, that is, scalefree network, has been studied by many researchers [3-23].

Compared with the ordinary differential equation (ODE) models (see [3-18] and references therein), more realistic models should be retarded functional differential equation (RFDE) models which can include some of the past states of these systems. Time delay plays an important role in the process of the epidemic spreading; for instance, the incubation period of the infectious diseases, the infection period of infective members, and the immunity period of the recovered individuals can be represented by time delays [24]. However, less attention has been paid to the epidemic models with time delays on heterogeneous network [19-22].

Zou et al. constructed a delayed SIR model without birth rate and death rate on scale-free network [19]. In the model, the discrete delay in model represents the incubation period in 2011. In 2014, Liu et al. also presented a delayed SIR model with birth rate and death rate on scale-free network. In this model, the discrete delay also represents the incubation period during which the infectious agents develop in the vector [21]. However, the assumption that the incubation period of an infective vector is determinate is somewhat idealized. And it is interesting to discuss the spreading of disease by using functional differential equation model with distributed delay [25]. Motivated by the work of Zou et al. [19] and Wang et al. [22], considering the fact the immune individual may become the susceptible individual [15], we will present a novel functional differential equation SIRS model with distributed delay on heterogeneous network in this paper to investigate the epidemic spreading, where the distributed delay represents the incubation period of an infective vector.

We consider the whole population and their contacts on network in which every individual is considered as a node in the network. Suppose the size of the network is a constant $N$ during the period of epidemic spreading; we also suppose that the degree of each node is time invariant; let $S_{k}(t)$, $I_{k}(t)$, and $R_{k}(t)$ be the relative density of susceptible nodes, infected nodes, and recovered nodes of connectivity $k$ at time $t$, respectively, where $k=m, m+1, \ldots, n$ in which $m$ and $n$ are the minimum and maximum number of contact each node, respectively.

In the process of the epidemic propagation via vector (such as mosquito), when a susceptible vector is infected by an infected nodes, there is a delay $\mu$ during which the 
infectious agents develop in the vector, and infected vector becomes itself infectious after the delay. At the same time, the vector's usual activities are in a limited range; that is, if a vector is infected by an infected node, its usual activities are in the vicinity of the infected node. Furthermore, if the vector population size is large enough, we can suppose that the number of the infectious vector population in the vicinity of the infected nodes with degree $k(k=1,2, \ldots, n)$ at any time $t$ is simply proportional to the number of the infected nodes with degree $k$ at time $t-\mu[25,26]$. Let the kernel function $f(u)$ denote the probability that an susceptible vector who is infected at time $t-\mu$ and becomes infective at time $t$. Meanwhile, let $\lambda(k)$ be the correlated ( $k$-dependent) infection rate such as $\lambda k$ and $\lambda c(k)$ [11]. The susceptible nodes may acquire temporary immunity and the removal rate from the susceptible nodes to the recovered nodes is given by $\delta$. And $\mu$ is removal rate from the recovered nodes to the susceptible nodes because the recovered nodes lose the temporary immunity. In addition, the infected nodes are cured with rate $r$. The dynamical equations for the density $S_{k}(t), I_{k}(t)$, and $R_{k}(t)$, at the mean-field level, satisfy the following set of functional differential equations when $t>0$ :

$$
\begin{aligned}
\dot{S}_{k}(t)= & -\lambda(k) S_{k}(t) \int_{0}^{+\infty} \Theta(t-u) f(u) d u-\delta S_{k}(t) \\
& +\mu R_{k}(t) \\
\dot{I}_{k}(t)= & \lambda(k) S_{k}(t) \int_{0}^{+\infty} \Theta(t-u) f(u) d u-r I_{k}(t), \\
\dot{R}_{k}(t)= & r I_{k}(t)-\mu R_{k}(t)+\delta S_{k}(t)
\end{aligned}
$$

with

$$
S_{k}(t)+I_{k}(t)+R_{k}(t)=1, \quad k=m, m+1, \ldots, n,
$$

due to the fact that the number of total nodes with degree $k$ is a constant $p(k) N$ during the period of epidemic spreading. The dynamics of $n$ groups of SIRS subsystems are coupled through the function $\Theta(t)$, which represents the probability that any given link points to an infected site. Assuming that the network has no degree correlations $[3,11]$, we have

$$
\Theta(t)=\frac{1}{\langle k\rangle} \sum_{k=m}^{n} \varphi(k) p(k) I_{k}(t),
$$

where $\langle k\rangle=\sum_{k} p(k) k$ stands for the average node degree and $\varphi(k)=a k^{\alpha} /\left(1+b k^{\alpha}\right)$ [7] $(0 \leq \alpha<1, a>0, b \geq 0)$ denotes an infected node with degree $k$ occupied edges which can transmit the disease. If $b \neq 0, \varphi(k)$ gradually become saturated with the increase of degree $k$, that is, $\lim _{t \rightarrow+\infty} \varphi(k)=$ $b / a$.

The kernel function $f(u)$ is nonnegative and continuous on $[0,+\infty)$ and satisfies

$$
\begin{gathered}
\int_{0}^{+\infty} f(u) d u=1, \\
\int_{0}^{+\infty} f(u) e^{\alpha \mu} d u<+\infty,
\end{gathered}
$$

where $\alpha$ is a positive number. And there are many types of kernel functions such as

(1) the gamma distribution $f(u)=\left(u^{n-1} /(n-1) ! b^{n}\right) e^{-u / b}$, where $b>0$ is a real number and $n>1$ is an integer, especially when $n=1, b=1$, and then $f(u)=e^{-u}$,

(2) the uniform distribution

$$
f(u)= \begin{cases}1 / h, & 0 \leq u \leq h \\ 0, & u>h,\end{cases}
$$

where $h>0$ is real number,

(3) the Delta-distribution $f(u)=\delta(u-\tau)$, where $\tau>0$ is real constant.

Define the following Banach space of fading memory type (see [27] and references therein):

$$
\begin{aligned}
\bar{C} & =\{\phi \in C((-\infty, 0], R): \phi(s) \\
& \cdot e^{\alpha s} \text { is uniformly continuous for } s \\
& \left.\in(-\infty, 0], \sup _{s \leq 0}|\phi(s)| e^{\alpha s}<+\infty\right\}
\end{aligned}
$$

with norm $\|\phi\|=\sup _{s \leq 0}|\phi(s)| e^{\alpha s}$, and let $\phi_{t} \in \bar{C}$ be such that $\phi_{t}(s)=\phi(t+s), s \in(-\infty, 0]$.

Consider system (1) in phase space $X=(\bar{C} \times \bar{C} \times$ $\bar{C})^{n-m+1}$. Standard theory of functional differential equation implies system (1) has a unique solution satisfying the initial conditions

$$
\begin{aligned}
& S_{k}(\theta)=\phi_{1 k}(s), \\
& I_{k}(\theta)=\phi_{2 k}(s), \\
& R_{k}(\theta)=\phi_{3 k}(s), \\
& \phi_{i k}(s) \geq 0, \\
& s \in(-\infty, 0], \phi_{i k}(0)>0, i=1,2,3, k=m, m+1, \ldots, n,
\end{aligned}
$$

where $\left(\phi_{1 m}(s), \phi_{2 m}(s), \phi_{3 m}(s), \ldots, \phi_{1 n}(s), \phi_{2 n}(s), \phi_{3 n}(s)\right) \in X$.

It can be verified that solutions of system (1) in $X$ with initial conditions above remain positive for all $t \geq 0$.

The rest of this paper is organized as follows. The dynamical behaviors of the SIRS model with distributed delay are discussed in Section 2. Numerical simulations and discussions are offered to demonstrate the main results in Section 3. 


\section{Dynamical Behaviors of the Model}

Since $S_{k}(t)+I_{k}(t)+R_{k}(t)=1$, system (1) is equivalent to the following system (8):

$$
\begin{aligned}
\dot{S}_{k}(t)= & -\lambda(k) S_{k}(t) \int_{0}^{+\infty} \Theta(t-u) f(u) d u-\delta S_{k}(t) \\
& +\mu\left(1-S_{k}(t)-I_{k}(t)\right), \\
\dot{I}_{k}(t)= & \lambda(k) S_{k}(t) \int_{0}^{+\infty} \Theta(t-u) f(u) d u-r I_{k}(t) .
\end{aligned}
$$

Thus we only discuss system (8) if we want to discuss the dynamical behaviors of system (1).

Denote

$$
R_{0}=\frac{\mu}{(\mu+\delta) r} \frac{\langle\lambda(k) \varphi(k)\rangle}{\langle k\rangle}
$$

where $\langle f(k)\rangle=\sum_{k} f(k) p(k)$ in which $f(k)$ is a function.

Note that we can obtain from the first equation of system (8) that

$$
\dot{S}_{k}(t) \leq \mu-(\mu+\delta) S_{k}(t) .
$$

By the standard comparison theorem in the theory of differential equations, we have

$$
\lim _{t \rightarrow+\infty} \sup S_{k}(t) \leq \frac{\mu}{\mu+\delta} .
$$

Hence we know

$$
\begin{aligned}
D_{0} & =\left\{\left(S_{m}, I_{m}, \ldots, S_{n}, I_{n}\right) \in R_{+}^{2(n-m+1)} \mid 0<S_{k}, I_{k}, S_{k}\right. \\
& \left.+I_{k} \leq 1,0 \leq S_{k} \leq \frac{\mu}{\mu+\delta}\right\}
\end{aligned}
$$

is positively invariant with respect to system (8), and every forward orbit in $R_{+}^{2(n-m+1)}$ eventually enters $D_{0}$.

Theorem 1. System (8) has always a disease-free equilibrium $E_{0}(\mu /(\mu+\delta), \ldots, \mu /(\mu+\delta), 0, \ldots, 0)$. System (8) has a unique endemic equilibrium $E_{*}\left(S_{m}^{*}, S_{m+1}^{*}, \ldots, S_{n}^{*}, I_{m}^{*}, I_{m+1}^{*}, \ldots, I_{n}^{*}\right)$ when $R_{0}>1$.

Proof. Obviously, the disease-free equilibrium $E_{0}$ of system (8) always exists. Now we discuss the existence of the endemic equilibrium of system (8). Combined with $\int_{0}^{+\infty} f(u) d u=1$, it is easy to know that the equilibrium $E_{*}$ satisfies

$$
\begin{aligned}
-\lambda(k) S_{k}^{*} \Theta^{*}-\delta S_{k}^{*}+\mu\left(1-S_{k}^{*}-I_{k}^{*}\right) & =0, \\
\lambda(k) S_{k}^{*} \Theta^{*}-r I_{k}^{*} & =0,
\end{aligned}
$$

where

$$
\Theta^{*}=\frac{1}{\langle k\rangle} \sum_{k} \varphi(k) p(k) I_{k}^{*}
$$

From (13), we obtain that

$$
I_{k}^{*}=\frac{\lambda(k) \mu \Theta^{*}}{(\mu+\delta) r+\lambda(k)(r+\mu) \Theta^{*}} .
$$

Substituting it into (14), we obtain the self-consistency equality

$$
\Theta^{*}=\frac{1}{\langle k\rangle} \sum_{k} \varphi(k) p(k) \frac{\lambda(k) \mu \Theta^{*}}{(\mu+\delta) r+\lambda(k)(r+\mu) \Theta^{*}},
$$

and it can be verified that (15) has a unique positive solution when $R_{0}>1$ using the same proof as for Theorem 1 in [21]; consequently, system (8) has a unique endemic equilibrium $E_{*}$ since (13) and (15) hold.

Theorem 2. If $R_{0}<1$, the disease-free equilibrium $E_{0}$ of system (8) is globally attractive.

Proof. Obviously, we need only discuss global attractiveness of system (8) in $D_{0}$.

Consider the following Lyapunov function

$$
V(t)=\frac{1}{2} V_{1}^{2}(t)+\frac{R_{0} r}{2} V_{2}(t)
$$

where

$$
\begin{aligned}
& V_{1}(t)=\int_{0}^{+\infty} \Theta^{2}(t-u) f(u) d u, \\
& V_{2}(t)=\int_{0}^{+\infty} f(u) \int_{t-2 u}^{t-u} \Theta^{2}(s) d s d u .
\end{aligned}
$$

Calculating the derivative of $V(t)$ along solution of (8), for $t>T_{1}$, we get

$$
\begin{aligned}
& \left.\dot{V}(t)\right|_{(9)}=\int_{0}^{+\infty} \Theta(t-u)\left(\frac{1}{\langle k\rangle} \sum_{k} \phi(k) p(k)(\lambda(k)\right. \\
& \left.\left.\cdot S_{k}(t) \int_{0}^{+\infty} \Theta(t-2 u) f(u) d u-r I_{k}(t-u)\right)\right) d u \\
& +\frac{R_{0} r}{2}\left(V_{1}(t)-\int_{0}^{+\infty} f(u) \Theta^{2}(t-2 u) d u\right) \\
& \quad \leq \int_{0}^{+\infty} \Theta(t-u)\left(\frac{1}{\langle k\rangle} \sum_{k} \phi(k) p(k)\right. \\
& \cdot\left(\lambda(k) \frac{\mu}{\mu+\delta} \int_{0}^{+\infty} \Theta(t-2 u) f(u) d u\right. \\
& \left.\left.-r I_{k}(t-u)\right)\right) d u+\frac{R_{0} r}{2}\left(V_{1}(t)\right. \\
& \left.-\int_{0}^{+\infty} f(u) \Theta^{2}(t-2 u) d u\right)=R_{0} r \int_{0}^{+\infty} \Theta(t-u)
\end{aligned}
$$




$$
\begin{aligned}
& \cdot \Theta(t-2 u) f(u) d u-r V_{1}(t) \\
& +\frac{R_{0} r}{2}\left(\int_{0}^{+\infty} f(u) \Theta^{2}(t-u) d u-\int_{0}^{+\infty} f(u) \Theta^{2}(t\right. \\
& -2 u) d u) \leq \frac{R_{0} r}{2} \int_{0}^{+\infty}\left(\Theta^{2}(t-u)+\Theta^{2}(t\right. \\
& -2 u)) f(u) d u-r V_{1}(t)+\frac{R_{0} r}{2}\left(\int_{0}^{+\infty} f(u)\right. \\
& \left.\cdot \Theta^{2}(t-u) d u-\int_{0}^{+\infty} \Theta^{2}(t-2 u) d u\right) \\
& =r\left(R_{0}-1\right) V_{1}(t) .
\end{aligned}
$$

Thus $\left.\dot{V}(t)\right|_{(9)} \leq 0$ when $R_{0}<1$, and $\left.\dot{V}(t)\right|_{(9)}=0$ if and only if $V_{1}(t)=0$. Note that the fact $\dot{V}(t)$ means $I_{k}=0$; moreover, $\lim _{t \rightarrow+\infty} S_{k}(t)=\mu /(\mu+\delta)$; the largest invariant set of $\left.\dot{V}(t)\right|_{(9)}=0$ is a singleton $E_{0}$. Hence the disease-free equilibrium $E_{0}$ is globally attractive when $R_{0}<1$ according to the LaSalle Invariance Principle [28, Chapter 2, Theorem 5.3].

Lemma 3 (see [28, p273-280]). Let $X$ be a complete metric space, $X=X^{0} \cup \partial X^{0}$, where $\partial X^{0}$, assumed to be nonempty, is the boundary of $X^{0}$. Assume the $C^{0}$-semigroup $T(t)$ on $X$ satisfies $T(x): X^{0} \rightarrow X^{0}, T(x): \partial X^{0} \rightarrow \partial X^{0}$ and

(i) there is a $t_{0}$ such that $T(t)$ is compact for $t>t_{0}$;

(ii) $T(t)$ is point dissipative in $X$;

(iii) $\widetilde{A}_{\partial}$ is isolated and has an acyclic covering $M$.

Then $T(t)$ is uniformly persistent if and only if, for each $M_{i} \in$ $M$,

$$
W^{s}\left(M_{i}\right) \cap X^{0}=\emptyset,
$$

where $\widetilde{A}_{\partial}=\bigcup_{x \in A_{\partial}} \omega(x)$, and $\omega(x)$ is the omega limit set of $T(x)$ through $x$, and $A_{\partial}$ is global attractor of $T_{\partial}(t)$ in $\partial X^{0}$ in which $T_{\partial}(t)=\left.T(t)\right|_{\partial X^{0}}$.

Theorem 4. For system (8), if $R_{0}>1$, the disease-free equilibrium $E_{0}$ is unstable, and the disease is uniformly persistent; that is, there exists a positive constant $\epsilon$ such that $\lim _{t \rightarrow+\infty} \inf I_{k}(t)>\epsilon, k=m, m+1, \ldots, n$.

Proof. Denote

$$
\begin{aligned}
X & =\left\{(\bar{S}, \bar{\psi}): \psi_{k}(\theta) \geq 0, \forall \theta \in(-\infty, 0], k=m, m\right. \\
& +1, \ldots, n\}, \\
X^{0} & =\left\{(\bar{S}, \bar{\psi}): \psi_{k}(\theta)>0, \text { for some } \theta \in(-\infty, 0], k\right. \\
& =m, m+1, \ldots, n\},
\end{aligned}
$$

and consequently,

$$
\begin{aligned}
\partial X^{0} & =\frac{X}{X^{0}}=\left\{(\bar{S}, \bar{\psi}): \psi_{i}(\theta)=0, \forall \theta \in(-\infty, 0], i\right. \\
& \in\{m, m+1, \ldots, n\}\},
\end{aligned}
$$

where $(\bar{S}, \bar{\psi})=\left(S_{m}, S_{m+1}, \ldots, S_{n}, \psi_{m}, \psi_{m+1}, \ldots, \psi_{n}\right)$.

Let $\left(S_{m}(t), \ldots, S_{n}(t), I_{m}(t), \ldots, I_{n}(t)\right)=\left(S_{m}(t, \omega), \ldots\right.$, $\left.S_{n}(t, \omega), I_{m}(t, \omega), \ldots, I_{n}(t, \omega)\right)$ be the solution of (8) with initial function $\omega=\left(\phi_{1 m}(s), \ldots, \phi_{1 n}(s), \phi_{2 m}(s), \ldots, \phi_{2 n}(s)\right)$ and

$$
\begin{aligned}
& T(t)(\omega)(\theta)=\left(S_{m}(t+\theta, \omega), \ldots, S_{n}(t+\theta, \omega),\right. \\
& \left.\quad I_{m}(t+\theta, \omega), \ldots, I_{n}(t+\theta, \omega)\right), \quad \theta \in(-\infty, 0] .
\end{aligned}
$$

Obviously, $X$ and $X^{0}$ are positively invariant set for $T(t) . T(t)$ is completely continuous for $t>0$. Also, it follows from $0<S_{k}, I_{k} \leq 1$ for $t>0$ that $T(t)$ is point dissipative. $E_{0}$ is the unique equilibrium of system (8) on $\partial X^{0}=X / X^{0}$ and it is globally stable on $\partial X^{0}=X / X^{0}, \widetilde{A}_{\partial}=\left\{E_{0}\right\}$, and $E_{0}$ is isolated and acyclic. Finally, the proof will be done if we prove $W^{s}\left(E_{0}\right) \cap X^{0}=\emptyset$, where $W^{s}\left(E_{0}\right)$ is the stable manifold of $E_{0}$. Suppose it is not true; then there exists a solution $(\bar{S}, \bar{I})$ in $X^{0}$ such that

$$
\begin{aligned}
\lim _{t \rightarrow+\infty} S_{k}(t) & =\frac{\mu}{\mu+\delta}, \\
I_{k}(t) & =0,
\end{aligned}
$$

$$
k=m, m+1, \ldots, n \text {. }
$$

Since $R_{0}>1$, we may choose $0<\eta<1$ such that $(\mu(1-$ $\eta) /(\lambda(k) \eta+\mu+\delta) r)(\langle\lambda(k) \varphi(k)\rangle /\langle k\rangle)>1$. At the same time, there exists a $T_{2}>0$ and $0<\epsilon<\eta$ such that $0 \leq I_{k}(t)<\eta$ for $t>T_{2}$ due to $\lim _{t \rightarrow+\infty} \inf I_{k}=0$.

When $t>T_{2}$, we obtain from the first equation of system (8) that

$$
\begin{aligned}
\dot{S}_{k}(t) & >-\lambda(k) S_{k} \eta+\mu\left(1-S_{k}(t)-\eta\right)-\delta S_{k}(t) \\
& =\mu(1-\eta)-(\lambda(k) \eta+\mu+\delta) S_{k}(t) .
\end{aligned}
$$

Hence there exist a $T_{3}>T_{2}$ such that the following equality holds when $t>T_{2}$ :

$$
S_{k}(t) \geq \frac{\mu(1-\eta)}{\lambda(k) \eta+\mu+\delta} .
$$

For $t>T_{2}$, we have from (3) and (26) that

$$
\begin{gathered}
\dot{\Theta}(t)=\frac{1}{\langle k\rangle} \sum_{k} \varphi(k) p(k) \dot{I}_{k}(t)=\frac{1}{\langle k\rangle} \sum_{k} \varphi(k) p(k) \\
\cdot\left[\lambda(k) S_{k}(t) \int_{0}^{+\infty} \Theta(t-\tau) f(u) d u-r I_{k}(t)\right] \\
\geq \frac{\mu(1-\eta)}{\lambda(k) \eta+\mu+\delta} \frac{\langle\lambda(k) \varphi(k)\rangle}{\langle k\rangle} \\
\quad \int_{0}^{+\infty} \Theta(t-\tau) f(u) d u-r \Theta(t) .
\end{gathered}
$$


By $(\mu(1-\eta) /(\lambda(k) \eta+\mu+\delta) r)(\langle\lambda(k) \varphi(k)\rangle /\langle k\rangle)>1$ and the comparison principle furthermore, it is easy to see that $\lim _{t \rightarrow+\infty} \Theta(t)=+\infty$, contradicting $\lim _{t \rightarrow+\infty} \Theta(t)=$ 0 as $\lim _{t \rightarrow+\infty} I_{k}(t)=0$. Hence $\lim _{t \rightarrow+\infty} \inf \Theta(t) \neq 0$; moreover, there exists $k_{0} \in\{m, m+1, \ldots, n\}$ such that $\lim _{t \rightarrow+\infty} \inf I_{k_{0}}(t) \neq 0$, contradicting $\lim _{t \rightarrow+\infty} \inf I_{k}(t)=0$, $k=m, m+1, \ldots, n$.

Hence, the infection is uniformly persistent according to Lemma 3; that is, there exists a $\epsilon$ is a positive constant such that $\lim _{t \rightarrow \infty} \inf I_{k}>\epsilon$, and the disease-free equilibrium $E_{0}$ is unstable accordingly. This completes the proof.

At last, let us discuss the global stability of the endemic equilibrium of system (8) by constructing suitable Lyapunov function.

Theorem 5. If $R_{0}>1, \delta<r$ and $I_{k}^{*}<\mu /(\mu+\delta)(\delta / r), k=$ $m, m+1, \ldots, n$, the endemic equilibrium $E_{*}$ of system (8) is globally asymptotically attractive.

Proof. For convenience, we still discuss system (1). According to (13) and $S_{k}(t)+I_{k}(t)+R_{k}(t)=1, k=m, m+1, \ldots, n$, we know

$$
\begin{aligned}
\widetilde{D}_{0} & =\left\{\left(S_{m}, I_{m}, R_{m}, \ldots, S_{n}, I_{n}, R_{n}\right) \in R_{+}^{2(n-m+1)} \mid 0\right. \\
& \left.<S_{k}, I_{k}, R_{k}, S_{k}+I_{k}+R_{n}=1,0 \leq S_{k} \leq \frac{\mu}{\mu+\delta}\right\}
\end{aligned}
$$

is positively invariant with respect to system (8), and every forward orbit in $R_{+}^{3(n-m+1)}$ eventually enters $\widetilde{D}_{0}$.

Thus we just need to discuss the global attractiveness of system (1) in $\widetilde{D}_{0}$.

Denote $R_{k}^{*}=1-S_{k}^{*}-I_{k}^{*}$, and then $E^{*}\left(S_{m}^{*}, I_{m}^{*}, R_{m}^{*}, S_{m+1}^{*}\right.$, $\left.I_{m+1}^{*}, R_{m+1}^{*}, \ldots, S_{n}^{*}, I_{n}^{*}, R_{n}^{*},\right)$ is the endemic equilibrium of system (1). System (1) may be rewritten as follows:

$$
\begin{aligned}
& \dot{S}_{k}(t)=-\sum_{l=m}^{n} \beta_{k l} S_{k}(t) \\
& \cdot \int_{0}^{+\infty} I_{l}(t-u) f(u) d u-\delta S_{k}(t)+\mu R_{k}(t), \\
& \dot{I}_{k}(t)=\sum_{l=m}^{n} \beta_{k l} S_{k}(t) \int_{0}^{+\infty} I_{l}(t-u) f(u) d u-r I_{k}(t), \\
& \dot{R}_{k}(t)=r-(\mu+r) R_{k}(t)-(r-\delta) S_{k}(t),
\end{aligned}
$$

where $\beta_{k l}=\lambda(l) \varphi(l) p(l) /\langle k\rangle, l=m, m+1, \ldots, n$.

Note that the endemic equilibrium of system (1) satisfies

$$
-\sum_{l=m}^{n} \beta_{k l} S_{k}^{*} I_{l}^{*}-\delta S_{k}^{*}+\mu R_{k}^{*}=0
$$

$$
\begin{aligned}
& \sum_{l=m}^{n} \beta_{k l} S_{k}^{*} I_{l}^{*} \\
& \quad=r I_{k}^{*}\left(\text { or } \sum_{l=m}^{n} \beta_{k l} S_{k}^{*} \int_{0}^{+\infty} I_{l}^{*} f(u) d u=r I_{k}^{*}\right), \\
& (\mu+r) R_{k}^{*}+(\delta-r) S_{k}^{*}=r .
\end{aligned}
$$

We have from (29) and (30) that

$$
\begin{aligned}
\dot{S}_{k}(t)= & -\sum_{l=m}^{n} \beta_{k l} S_{k}(t) \int_{0}^{+\infty} I_{l}(t-u) f(u) d u \\
& -\sum_{l=m}^{n} \beta_{k l} S_{k}^{*} \int_{0}^{+\infty} I_{l}^{*} f(u) d u \\
& -\delta\left(S_{k}(t)-S_{k}^{*}\right)+\mu\left(R_{k}(t)-R_{k}^{*}\right), \\
\dot{I}_{k}(t)= & \sum_{l=m}^{n} \beta_{k l} S_{k}(t) \int_{0}^{+\infty} I_{l}(t-u) f(u) d u \\
& -\frac{1}{I_{k}^{*}}\left(\sum_{l=m}^{n} \beta_{k l} S_{k}^{*} \int_{0}^{+\infty} I_{l}^{*} f(u) d u\right) I_{k}(t), \\
\dot{R}_{k}(t)= & -(\mu+r)\left(R_{k}(t)-R_{k}^{*}\right) \\
& +(\delta-r)\left(S_{k}(t)-S_{k}^{*}\right) .
\end{aligned}
$$

Let us consider

$$
\begin{aligned}
V_{k}(t)=S_{k}(t)-S_{k}^{*}-S_{k}^{*} \ln \frac{S_{k}(t)}{S_{k}^{*}}+I_{k}(t)-I_{k}^{*}-I_{k}^{*} \\
\qquad \cdot \ln \frac{I_{k}(t)}{I_{k}^{*}}+\sum_{l=m}^{n} \beta_{k l} S_{k}^{*} \int_{0}^{+\infty} f(u) \\
\quad \cdot \int_{t-u}^{t}\left(I_{l}(s)-I_{l}^{*}-I_{l}^{*} \ln \frac{I_{l}(s)}{I_{l}^{*}}\right) d s d u \\
+\frac{\mu}{S_{k}^{*}(r-\delta)} \frac{\left(R_{k}(t)-R_{k}^{*}\right)^{2}}{2} .
\end{aligned}
$$

Calculating the derivative of $V_{k}(t)$ along solution of (31), we get

$$
\begin{gathered}
\left.\dot{V}_{k}(t)\right|_{(32)}=-\delta\left(1-\frac{S_{k}^{*}}{S_{k}}\right)\left(S_{k}-S_{k}^{*}\right)+\mu\left(1-\frac{S_{k}^{*}}{S_{k}}\right) \\
\cdot\left(R_{k}-R_{k}^{*}\right)+\sum_{l=m}^{n} \beta_{k l} S_{k}^{*} I_{l}^{*} \int_{0}^{+\infty}\left(2-\frac{S_{k}^{*}}{S_{k}}-\frac{I_{k}}{I_{k}^{*}}\right. \\
\left.-\frac{S_{k} I_{l}(t-\tau) I_{k}^{*}}{S_{k}^{*} I_{k} I_{l}^{*}}-\ln \frac{I_{l}}{I_{l}^{*}}+\ln \frac{I_{l}(t-\tau)}{I_{l}^{*}}+\frac{I_{l}}{I_{l}^{*}}\right)
\end{gathered}
$$




$$
\begin{aligned}
& \cdot f(u) d u+\mu\left(R_{k}-R_{k}^{*}\right)\left(1-\frac{S_{k}}{S_{k}^{*}}\right) \\
& -\frac{\mu(r+\mu)}{S_{k}^{*}(r-\delta)}\left(R_{k}-R_{k}^{*}\right)^{2} .
\end{aligned}
$$

Since

$$
\begin{aligned}
& \sum_{l=m}^{n} \beta_{k l} S_{k}^{*} I_{l}^{*} \int_{0}^{+\infty}\left(2-\frac{S_{k}^{*}}{S_{k}}-\frac{I_{k}}{I_{k}^{*}}-\frac{S_{k} I_{l}(t-u) I_{k}^{*}}{S_{k}^{*} I_{k} I_{l}^{*}}\right. \\
& \left.-\ln \frac{I_{l}}{I_{l}^{*}}+\ln \frac{I_{l}(t-u)}{I_{l}^{*}}+\frac{I_{l}}{I_{l}^{*}}\right) f(u) d u \\
& =\sum_{l=m}^{n} \beta_{k l} S_{k}^{*} I_{l}^{*}\left(H\left(\frac{I_{k}}{I_{k}^{*}}\right)-H\left(\frac{I_{l}}{I_{l}^{*}}\right)\right) \\
& -\sum_{l=m}^{n} \beta_{k l} S_{k}^{*} I_{l}^{*}\left(G\left(\frac{S_{k}^{*}}{S_{k}}\right)+G\left(\frac{S_{k}}{S_{k}^{*}}\right)\right)-\sum_{l=m}^{n} \beta_{k l} S_{k}^{*} I_{l}^{*} \\
& \cdot \int_{0}^{+\infty} G\left(\frac{S_{k} I_{l}(t-u) I_{k}^{*}}{S_{k}^{*} I_{k} I_{l}^{*}}\right) f(u) d u \\
& +\sum_{l=m}^{n} \beta_{k l} S_{k}^{*} I_{l}^{*} G\left(\frac{S_{k}}{S_{k}^{*}}\right) \\
& -\delta\left(1-\frac{S_{k}^{*}}{S_{k}}\right)\left(S_{k}-S_{k}^{*}\right)=-\delta S_{k}^{*}\left(G\left(\frac{S_{k}}{S_{k}^{*}}\right)\right. \\
& \left.+G\left(\frac{S_{k}^{*}}{S_{k}}\right)\right) \mu\left(1-\frac{S_{k}^{*}}{S_{k}}\right)\left(R_{k}-R_{k}^{*}\right)+\mu\left(R_{k}-R_{k}^{*}\right) \\
& \cdot\left(1-\frac{S_{k}}{S_{k}^{*}}\right)=-\mu\left(R_{k}-R_{k}^{*}\right)\left(G\left(\frac{S_{k}}{S_{k}^{*}}\right)\right. \\
& \left.+G\left(\frac{S_{k}^{*}}{S_{k}}\right)\right)=-\mu R_{k} G\left(\frac{S_{k}}{S_{k}^{*}}\right)-\mu R_{k} G\left(\frac{S_{k}^{*}}{S_{k}}\right) \\
& +\mu R_{k}^{*}\left(G\left(\frac{S_{k}}{S_{k}^{*}}\right)+G\left(\frac{S_{k}^{*}}{S_{k}}\right)\right)
\end{aligned}
$$

hold, where $H(a)=-a+\ln a$ and $G(a)=a-1-\ln a \geq 0$, we can obtain from (30), (33), and (34) that

$$
\begin{aligned}
\left.\dot{V}_{k}(t)\right|_{(32)} \leq & \sum_{l=m}^{n} \beta_{k l} S_{k}^{*} I_{l}^{*}\left(H\left(\frac{I_{k}}{I_{k}^{*}}\right)-H\left(\frac{I_{l}}{I_{l}^{*}}\right)\right) \\
& -\mu R_{k} G\left(\frac{S_{k}}{S_{k}^{*}}\right)+\sum_{l=m}^{n} \beta_{k l} S_{k}^{*} I_{l}^{*} G\left(\frac{S_{k}}{S_{k}^{*}}\right) \\
\leq & \sum_{l=m}^{n} \beta_{k l} S_{k}^{*} I_{l}^{*}\left(H\left(\frac{I_{k}}{I_{k}^{*}}\right)-H\left(\frac{I_{l}}{I_{l}^{*}}\right)\right) \\
& +\left(-\mu R_{k}+r I_{k}^{*}\right) G\left(\frac{S_{k}}{S_{k}^{*}}\right) .
\end{aligned}
$$

Furthermore, by $r>\delta$ and $S_{k}(t) \leq \mu /(\mu+\delta)$, we have from the last equation of system (31) that

$$
\begin{aligned}
\dot{R}_{k}(t) & \geq r-(\mu+r) R_{k}(t)-(r-\delta) \frac{\mu}{\mu+\delta} \\
& =(\mu+r)\left(\frac{\delta}{\mu+\delta}-R_{k}(t)\right)
\end{aligned}
$$

Since $I_{k}^{*}<\delta /(\mu+\delta)(\mu / r)$, we can take $\varepsilon=\delta /(\mu+\delta)-I_{k}^{*} r / \mu>$ 0 , and it follows from (42) that there exists a $T_{4}>T_{1}$ such that $R_{k}(t) \geq \delta /(\mu+\delta)-\varepsilon$ when $t>T_{4}$. Hence $-\mu R_{k}+r I_{k}^{*} \leq$ $-\mu(\delta /(\mu+\delta)-\varepsilon)+r I_{k}^{*}=0$ when $t>T_{4}$, that is,

$$
\left(-\mu R_{k}+r I_{k}^{*}\right) G\left(\frac{S_{k}}{S_{k}^{*}}\right) \leq 0 .
$$

In addition, the matrix

$$
\begin{aligned}
& \left(\beta_{k l} S_{k}^{*} I_{l}^{*}\right)_{(n-m+1) \times(n-m+1)} \\
& \quad=\left(\frac{\lambda(l) \varphi(l) p(l)}{\langle k\rangle} S_{k}^{*} I_{l}^{*}\right)_{(n-m+1) \times(n-m+1)}
\end{aligned}
$$

is irreducible, so the following matrix is irreducible:

$$
B=\left(\begin{array}{cccc}
\sum_{l \neq m} \beta_{m l} S_{m}^{*} I_{l}^{*} & -\beta_{m+1, m} S_{m+1}^{*} I_{m}^{*} & \cdots & -\beta_{n m} S_{n}^{*} I_{m}^{*} \\
-\beta_{m, m+1} S_{m}^{*} I_{m+1}^{*} & \sum_{l \neq m+1} \beta_{m+1, l} S_{m+1}^{*} I_{l}^{*} & \cdots & -\beta_{n, m+1} S_{n}^{*} I_{m+1}^{*} \\
\cdots & \cdots & \cdots & \cdots \\
-\beta_{m n} S_{m}^{*} I_{n}^{*} & -\beta_{m+1, n} S_{m+1}^{*} I_{n}^{*} & \cdots & \sum_{l \neq n} \beta_{n l} S_{n}^{*} I_{l}^{*}
\end{array}\right) .
$$


Hence there exists a positive vector $C=\left(c_{1}, c_{2}, \ldots, c_{n}\right)$ such that $B C=0$ in which $c_{k}$ is the cofactor of the $k$ th diagonal of $B, m \leq k \leq n$ [29, Lemma 2.1]. It follows from $B C=0$ that

$$
\sum_{l=m}^{n} c_{l} \beta_{l k} S_{l}^{*} I_{k}^{*}=c_{k} \sum_{l=1}^{n} \beta_{k l} S_{k}^{*} I_{l}^{*}, \quad k=m, m+1, \ldots, n,
$$

which leads to

$$
\begin{gathered}
\sum_{k=m}^{n} c_{k} \sum_{l=m}^{n} \beta_{k l} S_{l}^{*} I_{l}^{*} H\left(\frac{I_{k}}{I_{k}^{*}}\right)=\sum_{k=m}^{n} \sum_{l=m}^{n} c_{l} \beta_{l k} S_{l}^{*} I_{k}^{*} H\left(\frac{I_{k}}{I_{k}^{*}}\right) \\
=\sum_{k=m}^{n} \sum_{l=m}^{n} c_{k} \beta_{k l} S_{k}^{*} I_{l}^{*} H\left(\frac{I_{l}}{I_{l}^{*}}\right) \\
=\sum_{k=m}^{n} c_{k} \sum_{l=m}^{n} \beta_{k l} S_{k}^{*} I_{l}^{*} H\left(\frac{I_{l}}{I_{l}^{*}}\right)
\end{gathered}
$$

that is,

$$
\sum_{k=1}^{n} c_{k} \sum_{l=1}^{n} \beta_{k l} S_{k}^{*} I_{l}^{*}\left(H\left(\frac{I_{k}}{I_{k}^{*}}\right)-H\left(\frac{I_{l}}{I_{l}^{*}}\right)\right)=0 .
$$

Define a Lyapunov function

$$
V(t)=\sum_{k=1}^{n} c_{k} V_{k}(t)
$$

where $V_{k}(t)$ is defined by (32), and we have from (35), (37), and (42) that

$$
\left.\dot{V}(t)\right|_{(32)} \leq 0
$$

Moreover, $\left.\dot{V}(t)\right|_{(32)}=0$ if and only if $S_{k}=S_{k}^{*}, I_{k}=I_{k}^{*}$, $R_{k}=R_{k}^{*}$. Therefore, LaSalle Invariance Principle [28, Chapter 2 , Theorem 5.3] implies that the endemic equilibrium $E^{*}$ of system (1) is globally attractive when $R_{0}>1, \delta<r$ and $I_{k}^{*}<\mu /(\mu+\delta)(\delta / r), k=m, m+1, \ldots, n$. The proof is completed.

\section{Numerical Simulation and Discussion}

The basic reproductive number for system (8) (or (1)) is

$$
R_{0}=\frac{\mu}{(\mu+\delta) r} \frac{\langle\lambda(k) \varphi(k)\rangle}{\langle k\rangle} .
$$

The equilibrium $E_{0}$ is globally attractive and the infection eventually disappears when $R_{0}<1$, and the infection will always exist when $R_{0}>1$. Note that $R_{0}$ is irrelevant to the distributed delay.

Extensive numerical simulations are carried out on scalefree model to demonstrate the mentioned theorems above. The simulations are based on system (8) and a scale-free networks in which the degree distribution is $p(k)=C k^{-\gamma}$, and $C$ satisfies $\sum_{k=m}^{n} p(k)=1$. Supposing the network is finite one, the maximum connectivity $n$ of any node is related to the network age, measured as the number of nodes $N[3,7]$ :

$$
n=m N^{1 /(\gamma-1)} \text {. }
$$

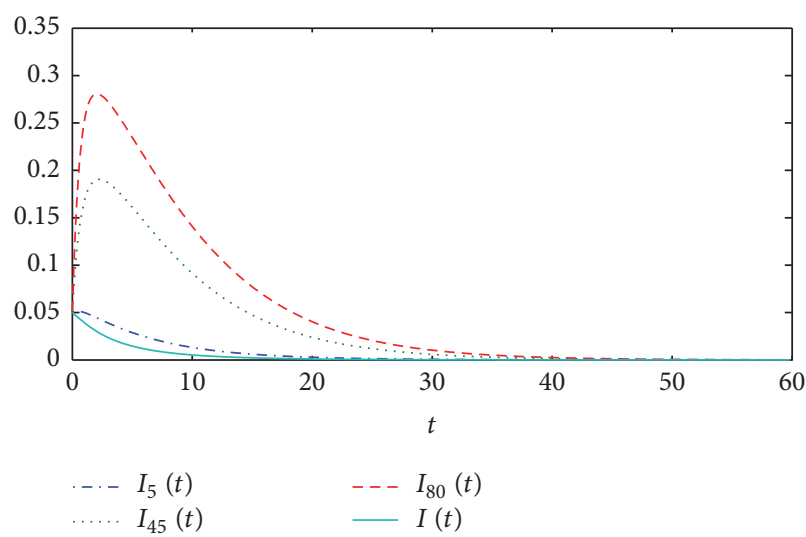

FIgURE 1: Dynamical behaviors of system (8) with $R_{0}=0.5547$.

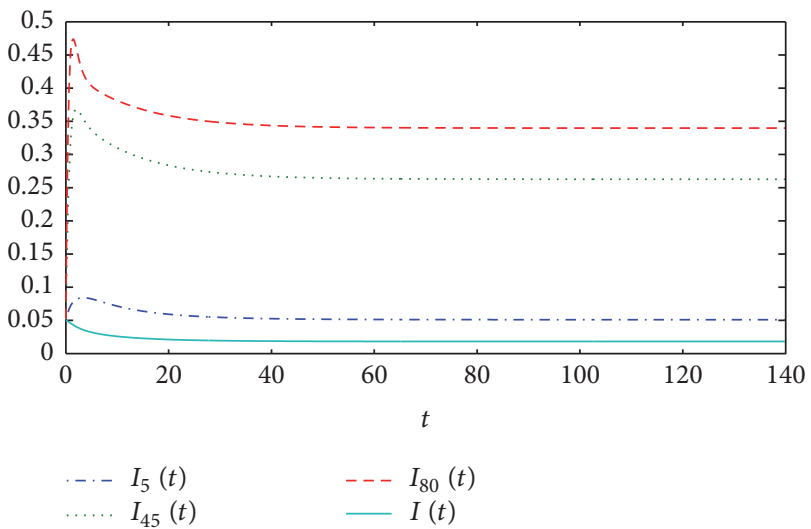

FIGURE 2: Dynamical behaviors of system (8) with $r=0.5>\delta=0.4$, $\max I_{k}^{*}=0.3668<\mu /(\mu+\delta)(\delta / r)=0.4800$ and $R_{0}=1.2944$.

Let $n=100$ and $m=1$ is a suitable assumption. Meanwhile, let $\phi(k)=a k^{\alpha} /\left(1+b k^{\alpha}\right)$ in which $a=0.5, \alpha=0.75, b=0.02$, and $\lambda(k)=\lambda k$. The initial functions are $I_{k}(s)=0.45, k=$ $2,3,4,5$ and $I_{k}=0, k \neq 2,3,4,5$ for $s \in(-\infty, 0]$.

Denote

$$
I(t)=\sum_{k} p(k) I_{k}(t)
$$

Obviously, $I(t)$ is the relative average density of the infected nodes.

Case 1. Let $\lambda=0.3, r=0.4, \delta=0.7, \mu=0.6, \gamma=2.5$, and $f(u)=e^{-u}$; we can obtain from $(45)$ that $R_{0}=0.5064<1$. Figure 1 shows the dynamical behaviors of system (8). The numerical simulation shows $\lim _{t \rightarrow+\infty} I(t)=0$, it follows that $\lim _{t \rightarrow+\infty} I_{k}(t)=0$, and the infection eventually disappears. The numerical result is consistent with Theorem 2 .

Case 2. Let $\lambda=0.7, r=0.5, \delta=0.4, \mu=0.6, \gamma=2.5$ and $f(u)=e^{-u}$, and $\delta<r$. We can obtain from (45) that $R_{0}=1.4596>1$ and $\max I_{k}^{*}=0.3668<\mu /(\mu+\delta)(\delta / r)=$ 0.4800 . Figure 2 shows the dynamic behaviors of system (8). The relative density $I_{k}(t)$ and the relative average density $I(t)$ converge to positive constant as $t \rightarrow+\infty$, respectively, and 


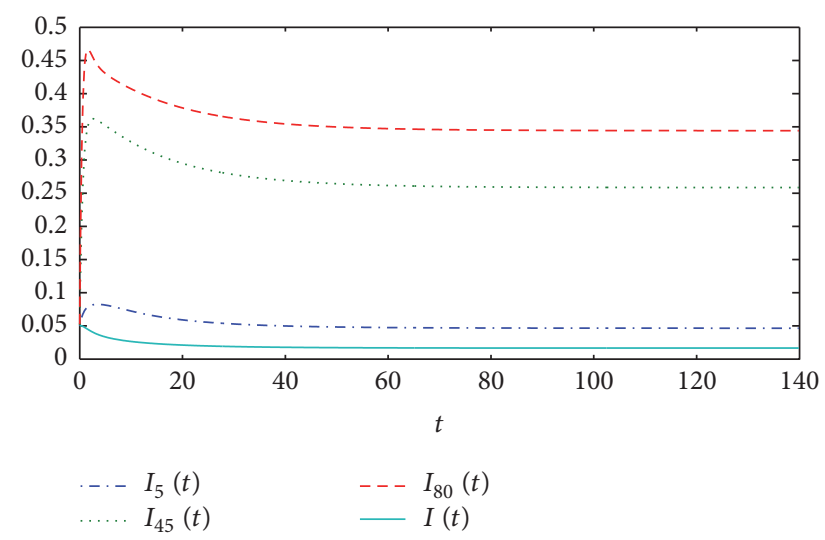

Figure 3: Dynamical behaviors of system (8) with $r=0.4<\delta=0.7$ and $R_{0}=1.3462$.

the infection is uniformly persistent. The numerical result is consistent with Theorems 4 and 5.

Moreover, let $\lambda=0.1, r=0.4, \delta=0.7, \mu=0.6$, $\gamma=2.5$ and $f(u)=e^{-u}$, and $\delta>r$. Figure 3 shows the dynamic behaviors of system (8). The parameters of system (8) do not satisfy Theorem 5 , but the relative density $I_{k}(t)$ and the relative average density $I(t)$ still converge to positive constant as $t \rightarrow+\infty$, respectively. Therefore, Theorem 5 has room for improvement.

\section{Competing Interests}

The authors declare that they have no competing interests.

\section{Acknowledgments}

The authors thank Professor Rui Xu with Shijiazhuang Mechanical Engineering College for his helpful suggestions. This research was supported by the Hebei Provincial Natural Science Foundation of China under Grant no. A2016506002 and the Innovation Foundation of Shijiazhuang Mechanical Engineering College under Grant no. YSCX1201.

\section{References}

[1] D. J. Watts and S. H. Strogatz, "Collective dynamics of small world networks," Nature, vol. 393, pp. 440-442, 1998.

[2] A.-L. Barabási and R. Albert, "Emergence of scaling in random networks," Science, vol. 286, no. 5439, pp. 509-512, 1999.

[3] R. Pastor-Satorras and A. Vespignani, "Epidemic dynamics in finite size scale-free networks," Physical Review E, vol. 65, no. 3, Article ID 035108, 2002.

[4] J. Balthrop, S. Forrest, M. E. J. Newman, and M. M. Williamson, "Technological networks and the spread of computer viruses," Science, vol. 304, no. 5670, pp. 527-529, 2004.

[5] R. Yang, B.-H. Wang, J. Ren et al., "Epidemic spreading on hererogeneous networks with identical infectivity," Physics Letters A, vol. 364, no. 3-4, pp. 189-193, 2007.

[6] C.-Y. Xia, Z.-X. Liu, Z.-Q. Chen, and Z.-Z. Yuan, "Spreading behavior of SIS model with non-uniform transmission on scale-free networks," Journal of China Universities of Posts and Telecommunications, vol. 16, no. 1, pp. 27-31, 2009.

[7] H. Zhang and X. Fu, "Spreading of epidemics on scale-free networks with nonlinear infectivity," Nonlinear Analysis. Theory, Methods \& Applications, vol. 70, no. 9, pp. 3273-3278, 2009.

[8] K. Li, M. Small, H. Zhang, and X. Fu, "Epidemic outbreaks on networks with effective contacts," Nonlinear Analysis. Real World Applications, vol. 11, no. 2, pp. 1017-1025, 2010.

[9] X. Fu, M. Small, D. M. Walker, and H. Zhang, "Epidemic dynamics on scale-free networks with piecewise linear infectivity and immunization," Physical Review E, vol. 77, no. 3, Article ID 036113, 2008.

[10] J.-p. Zhang and Z. Jin, "The analysis of an epidemic model on networks," Applied Mathematics and Computation, vol. 217, no. 17, pp. 7053-7064, 2011.

[11] G. Zhu, X. Fu, and G. Chen, "Global attractivity of a networkbased epidemics SIS model with nonlinearn infectivity," Communications in Nonlinear Science and Numerical Simulation, vol. 17, pp. 2588-2594, 2013.

[12] Y.-W. Gong, Y.-R. Song, and G.-P. Jiang, "Epidemic spreading in scale-free networks including the effect of individual vigilance," Chinese Physics B, vol. 21, no. 1, Article ID 010205, 2012.

[13] T. Li, Y. Wang, and Z.-H. Guan, "Spreading dynamics of a SIQRS epidemic model on scale-free networks," Communications in Nonlinear Science and Numerical Simulation, vol. 19, no. 3, pp. 686-692, 2014.

[14] J. Liu and T. Zhang, "Epidemic spreading of an SEIRS model in scale-free networks," Communications in Nonlinear Science and Numerical Simulation, vol. 16, no. 8, pp. 3375-3384, 2011.

[15] L. Chen and J. Sun, "Global stability and optimal control of an SIRS epidemic model on heterogeneous networks," Physica A. Statistical Mechanics and its Applications, vol. 410, pp. 196-204, 2014.

[16] R. Yu, K. Li, B. Chen, and D. Shi, "Dynamical analysis of an SIRS network model with direct immunization and infective vector," Advances in Difference Equations, vol. 2015, article no. 116, 2015.

[17] X. Xu and G. Chen, "The SIS model with time delay on complex networks," International Journal of Bifurcation and Chaos, vol. 19, pp. 623-662, 2009.

[18] C.-y. Xia, Z. Wang, J. Sanz, S. Meloni, and Y. Moreno, "Effects of delayed recovery and nonuniform transmission on the spreading of diseases in complex networks," Physica A. Statistical Mechanics and its Applications, vol. 392, no. 7, pp. 1577-1585, 2013.

[19] S. Zou, J. Wu, and Y. Chen, "Multiple epidemic waves in delayed susceptible-infected-recovered models on complex networks," Physical Review E-Statistical, Nonlinear, and Soft Matter Physics, vol. 83, no. 5, Article ID 056121, 2011.

[20] X. Liu and D.-J. Xu, "Analysis of SE $\tau$ IR $\omega$ S epidemic disease models with vertical transmission in complex networks," Acta Mathematicae Applicatae Sinica. English Series, vol. 28, no. 1, pp. 63-74, 2012.

[21] Q.-M. Liu, C.-S. Deng, and M.-C. Sun, "The analysis of an epidemic model with time delay on scale-free networks," Physica A. Statistical Mechanics and its Applications, vol. 410, pp. 79-87, 2014.

[22] J. Wang, J. Wang, M. Liu, and Y. Li, "Global stability analysis of an SIR epidemic model with demographics and time delay on networks," Physica A: Statistical Mechanics and Its Applications, vol. 410, pp. 268-275, 2014. 
[23] H. Kang and X. Fu, "Epidemic spreading and global stability of an SIS model with an infective vector on complex networks," Communications in Nonlinear Science and Numerical Simulation, vol. 27, no. 1-3, pp. 30-39, 2015.

[24] J. Zhen, Z. Ma, and M. Han, "Global stability of an SIRS epidemic model with delays," Acta Mathematica Scientia. Series B. English Edition, vol. 26, no. 2, pp. 291-306, 2006.

[25] Z. Ma and J. Li, Dynamical Modelling and Analysis of Epidemics, World Scientific, Singapore, 2009.

[26] E. Beretta, T. Hara, W. Ma, and Y. Takeuchi, "Global asymptotic stability of an SIR epidemic model with distributed time delay," Nonlinear Analysis, vol. 47, pp. 4107-4115, 2001.

[27] Y. Hino, S. Murakami, and T. Naito, Functional Differential equations with Infinite Delay, vol. 1473 of Lecture Notes in Mathematics, Springer, Berlin, Germany, 1991.

[28] Y. Kuang, Delay Differential Equations with Applications in Population Dynamics, Mathematics in Science and Engineering, Academic Press, Boston, Mass, USA, 1993.

[29] H. Guo, M. Y. Li, and Z. Shuai, "Global stability of the endemic equilibrium of multigroup SIR epidemic models," Canadian Applied Mathematics Quarterly, vol. 14, no. 3, pp. 259-284, 2006. 


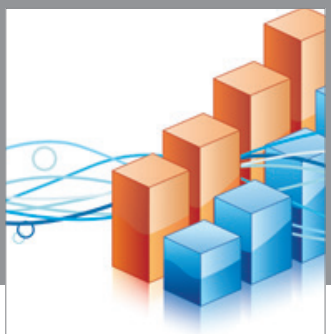

Advances in

Operations Research

vatem alat4

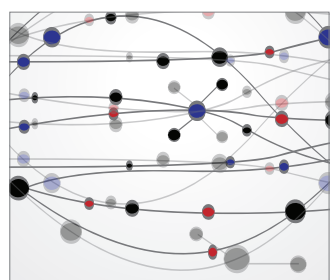

\section{The Scientific} World Journal
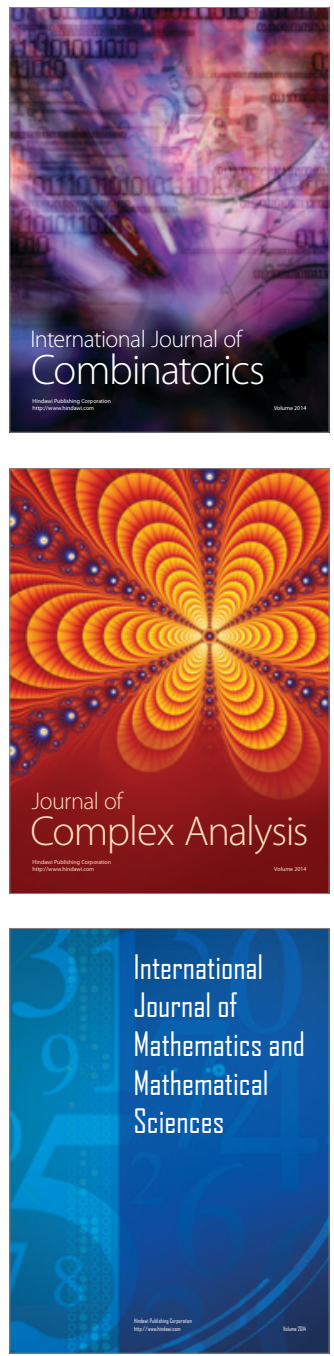
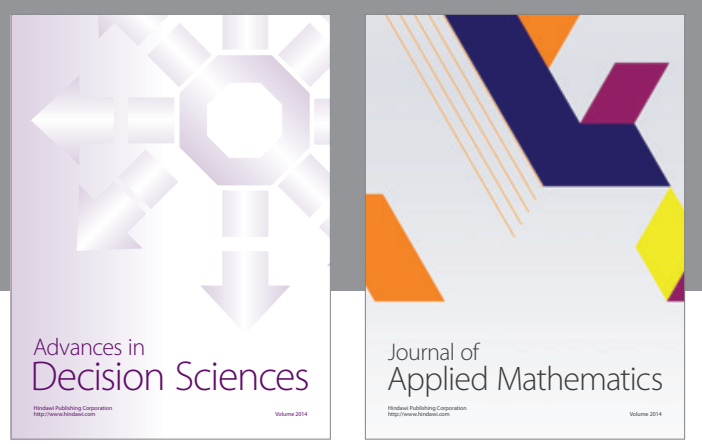

Algebra

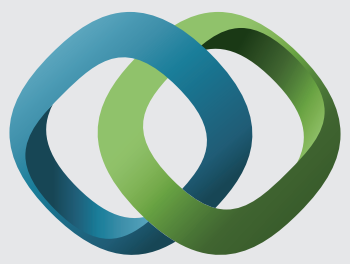

\section{Hindawi}

Submit your manuscripts at

https://www.hindawi.com
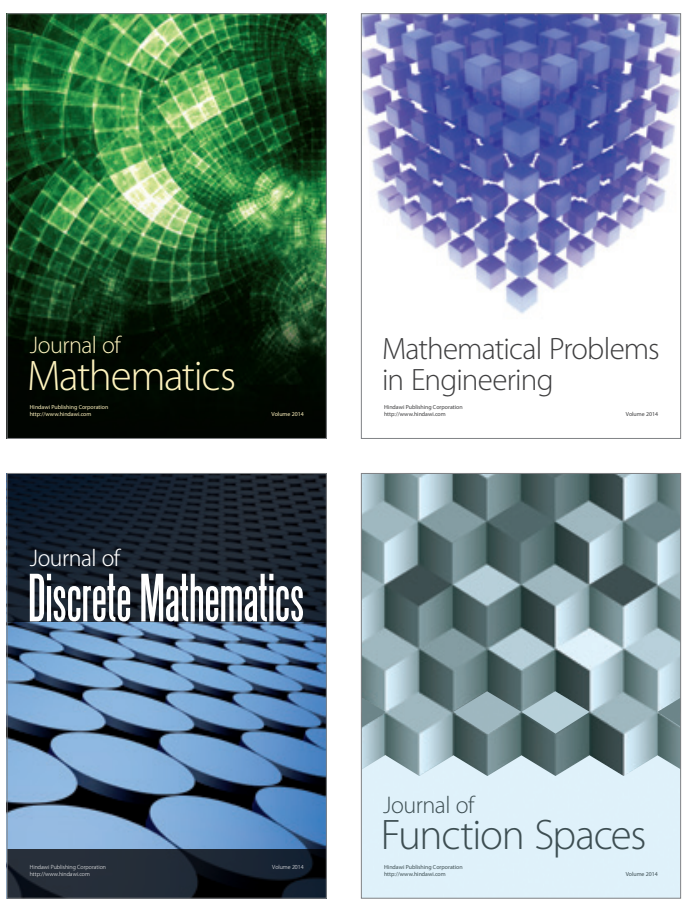

Mathematical Problems in Engineering
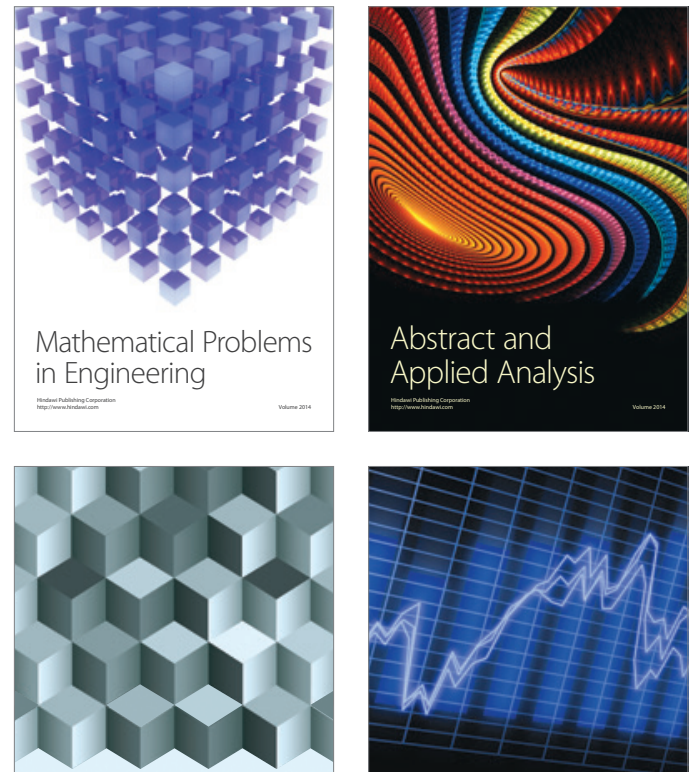

Journal of

Function Spaces

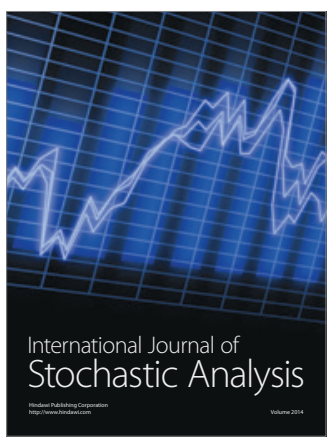

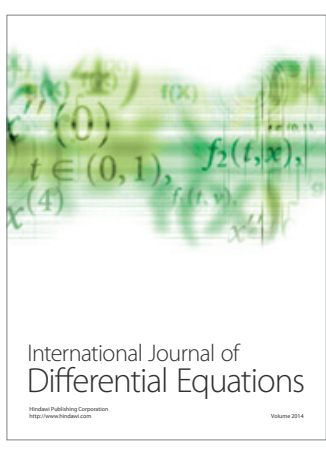
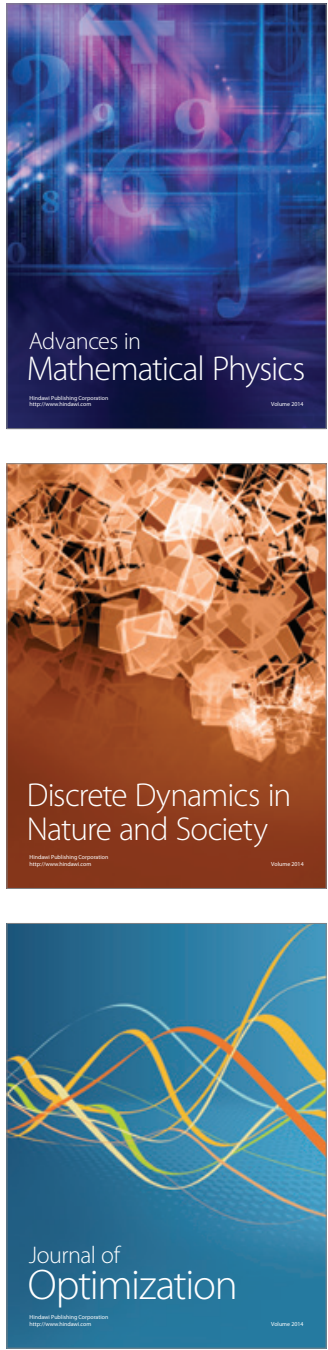